\title{
Towards Identification of Oxygen Point Defects by Means of Position Averaged CBED
}

\author{
R. dos Reis, C. Ophus, J. Ciston, P. Ercius, U. Dahmen \\ National Center for Electron Microscopy/Molecular Foundry, LBNL, Berkeley, CA 94720, USA
}

Point defects strongly affect the physical properties of materials and have a determinant impact on their performance. Extensive experimental research has been devoted to their characterization in previous decades. However, the understanding and control of point defects is limited and remains one of the challenging issues in the design of new materials. In this work, we explore the possibilities and limitations of detecting oxygen vacancy sites in Zinc Oxide $(\mathrm{ZnO})$ by means of Position Averaged CBED [1]. PACBED patterns are very sensitive to thickness variation and to small tilts and deformations of the unit cell, with the advantage of being independent of aberrations. In particular, $\mathrm{ZnO}$ nanostructures contain a large density of intrinsic defects, especially oxygen vacancies $\left(V_{O}\right)$, which play an important role in the green light emission. $\mathrm{V}_{\mathrm{O}}$ sites exhibit different local relaxations depending on their charge state [2]. $\mathrm{Zn}$ atoms surrounding the site in a neutral charge state $\left(\mathrm{V}_{\mathrm{O}}{ }^{\times}\right)$relax inward approximately $11 \%(1.952 \AA$ vs $1.733 \AA)$. On the other hand, a strong outward relaxation of about $19 \%$ is found for the charged vacancy $\left(\mathrm{V}_{\mathrm{O}}^{++}\right)$.

In order to evaluate the effect of vacancies on a PACBED pattern, Figure 1 presents multislice simulations for an ideal $\mathrm{ZnO}$ crystal, $\mathrm{ZnO}$ with $\mathrm{V}_{\mathrm{O}}{ }^{++}$-type vacancy, and with $\mathrm{V}_{\mathrm{O}}{ }^{\times}$-type vacancy. A layer thickness of $100 \AA$ ( $~ 30$ unit cells) was used for all cases, applying the atomic configuration shown in the first row of Fig. 1. A sublayer containing a single point defect replaced an ideal $\mathrm{ZnO}$ slab in the middle of the supercell. Figures 1 (a-c) show the results along the $\langle 10 \overline{1} 0\rangle$ zone axis. Figures $1(\mathrm{~d}-\mathrm{f})$ show the simulations along the $\langle 11 \overline{2} 0\rangle$ zone axis.

The local distortions caused by the presence of point defects can be noticed as a subtle intensity variation within the PACBED discs. To illustrate this effect, Figure 2 plots differential maps for the simulated PACBED patterns. It compares layers containing $\mathrm{V}_{\mathrm{O}}{ }^{\times}$and $\mathrm{V}_{\mathrm{O}}{ }^{++}$to an ideal $\mathrm{ZnO}$ layer along $\langle 10 \overline{1} 0\rangle$ (Fig 2(a-b)) and $\langle 11 \overline{2} 0\rangle$ (Fig. 2(c-d)), respectively. Differential maps highlight the characteristic features of each ideal case, otherwise unnoticeable. They also show that the range of intensity variation falls within $\pm 25 \%$ of the total PACBED intensity.

The feasibility of the method was tested by acquiring experimental data from $\mathrm{ZnO}$ nanowires as 4DSTEM datasets [3]. CBED patterns were recorded at many probe positions with millisecond dwell times by using a Gatan K2-IS direct electron detection camera installed on the TEAM I microscope [3]. Ideal PACBED patterns were constructed by averaging the probe images over each $\mathrm{ZnO}$ unit cell along the $\langle 10 \overline{1} 0\rangle$ zone axis. Figure 3(a) presents a pattern fitted from the central area of a simultaneously acquired HAADF image (Fig. 3(c)). A mean thickness of $220 \AA$ was determined by comparison with simulated patterns (Fig. 3(a) bottom). Figure 3(b) shows a differential map comparing the PACBED of a single unit cell (center) to that of its eight first neighbors. It is observed that the signal to noise ratio is sufficient to detect the variations within the patterns $( \pm 25 \%$ of the intensity). The presence of $O$ vacancies may be determined by refining the positions of the PACBED discs and by matching with simulated patterns. Detailed analysis is currently underway and the issues concerning this method will be addressed alongside the code development to improve the precision [4]. 
[1] JM LeBeau et al. Ultramicroscopy 110, p.118 (2010).

[2] P. Erhart et al. Phys. Rev. B 73, 205203 (2006).

[3] C. Ophus et al. Acta Cryst A70, C1455 (2014).

[4]Work at the NCEM/Molecular Foundry was supported by the Office of Science, Office of Basic Energy Sciences, of the U.S. Department of Energy under Contract No. DE-AC02-05CH11231. RR acknowledges the support of CAPES/BR foundation Process No. 1204713-9 and thanks Dr. D.L. Baptista and Dr. C.I.L. Sombrio for providing $\mathrm{ZnO}$ nanowires for this study.

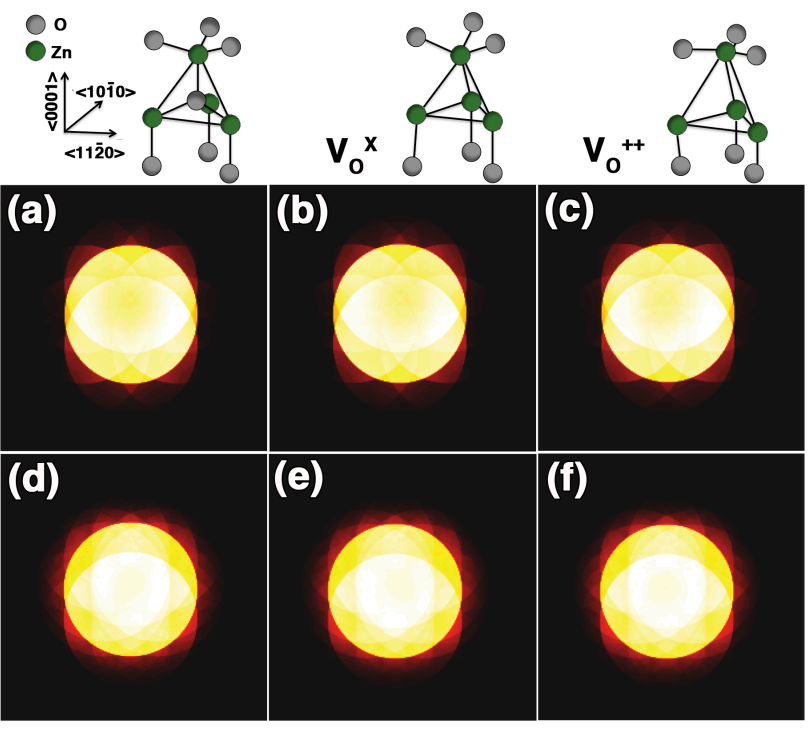

Figure 1: Simulated PACBED patterns using multislice for an ideal $\mathrm{ZnO}$, a sample containing a single $\mathrm{V}_{\mathrm{O}}{ }^{\times}$and, $\mathrm{V}_{\mathrm{O}}^{++}$, along $\langle 10 \overline{1} 0\rangle$ zone axis $(\mathrm{a}-\mathrm{c})$ and along $\langle 11 \overline{2} 0\rangle$ zone axis. The structural model for each type of defect is shown on the top row.
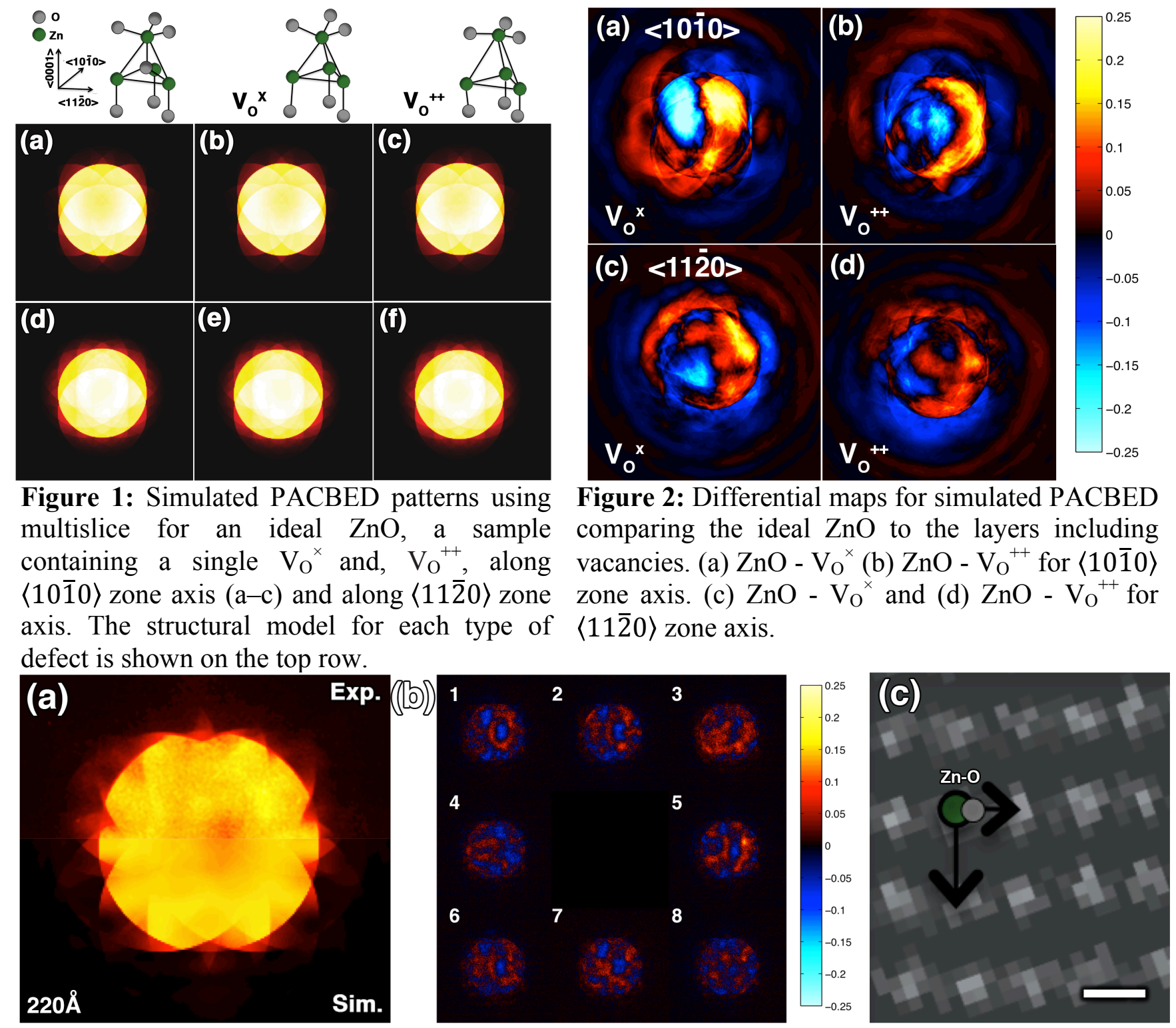

Figure 2: Differential maps for simulated PACBED comparing the ideal $\mathrm{ZnO}$ to the layers including vacancies. (a) $\mathrm{ZnO}-\mathrm{V}_{\mathrm{O}}{ }^{\times}$(b) $\mathrm{ZnO}-\mathrm{V}_{\mathrm{O}}^{++}$for $\langle 10 \overline{1} 0\rangle$ zone axis. (c) $\mathrm{ZnO}-\mathrm{V}_{\mathrm{O}}{ }^{\times}$and (d) $\mathrm{ZnO}-\mathrm{V}_{\mathrm{O}}{ }^{++}$for $\langle 11 \overline{2} 0\rangle$ zone axis.

Figure 3: (a) Experimental PACBED constructed from the central unit cell of HAADF image (c), alongside the simulated pattern with thickness of $220 \AA$. (b) Differential map comparing the central PACBED to eight first neighbors. (c) HAADF acquired simultaneously to the probe images and used to fit the unit cells along $\langle 10 \overline{1} 0\rangle$ zone axis (scale bar of $3 \AA$ ). 